\title{
Corrigenda:
}

\section{Hopf Bifurcation at Multiple Eigenvalues}

(Volume 69, 53-83 (1979))

\section{HANSJÖRG KIELHÖFER}

Page 60. In the eighth line from the bottom, insert $2 \pi$ after $=$.

Theorem 2.1 is in error as stated. To correct this, the last three sentences on page 69 should be replaced by:

We confine ourselves to the following case $(a=0)$ :

$$
a_{j+2}-a_{j 0}-c_{j}=0, j=0, \ldots, n-1 ; \quad a_{n+2}-a_{n}-c_{n} \neq 0,
$$

where $c_{0}=0$ and

$$
c_{j}=\sum_{n=0}^{j-1} \sum_{m=1}^{j-n} a_{n m} \sum_{v_{1}+\ldots+v_{m}=j-n} d_{v_{1}} \ldots d_{v_{m}}, \quad j \geqq 1,
$$

and where the $d_{j}, j \geqq 1$, are recursively defined by

$$
\begin{aligned}
d_{1} & =b, \quad d_{2}=b_{2}-b_{00}, \\
d_{j+2} & =b_{j+2}-b_{j 0}-\sum_{n=0}^{j-1} \sum_{m=1}^{j-n} b_{n m} \sum_{v_{1}+\ldots+v_{m}=j-n} d_{v_{1}} \ldots d_{v_{m}}, j \geqq 1 .
\end{aligned}
$$

If $n$ is even, we assume that

(2.15) $\operatorname{sign}\left(a_{n+2}-a_{n 0}-c_{n}\right)=\operatorname{sign} a_{1}$

but put no restriction on the sign of $\lambda$. If $n$ is odd, we suppose only that

$$
\operatorname{sign} \lambda=\operatorname{sign} a_{1}\left(a_{n+2}-a_{n 0}-c_{n}\right) .
$$

Page 71. Corollary 2.3 is wrong in general. It is true if we assume in addition that $P B=B P$, where $P u=2 \pi\left[\left(u, \varphi_{1}^{*}\right) \varphi_{1}+\left(u, \bar{\varphi}_{1}^{*}\right) \bar{\varphi}_{1}^{*}\right]$.

The correction of Theorem 2.1 has no influence on the validity of Theorem 2.4 and 3.2. For Theorem 2.4 this is clear from the following change, whereas for Theorem 3.2 observe that the coefficients $a_{n m}=0$ for all $n$ and $m$. 
Page 72. Replace the tenth line from the bottom by: $k \geqq 0$.

It can now be shown that $\delta_{1}=\ldots=\delta_{n-1}=0$ implies $a_{j k}=0$ for $0 \leqq j \leqq n-2$,

Page 81. Replace the fifth line by:

$$
\|F(u)\| \leqq c_{19}\left\|A_{0}^{\beta} u\right\|^{3}, \quad \beta>1 / 2,
$$

Institut für Angewandte Mathematik und Statistik Am Hubland 8700 Würzburg W. Germany

(Received May 22, 1979) 